(C) 2019 IEEE. Personal use of this material is permitted. Permission from IEEE must be obtained for all other uses, in any current or future media, including reprinting/republishing this material for advertising or promotional purposes, creating new collective works, for resale or redistribution to servers or lists, or reuse of any copyrighted component of this work in other works. 


\title{
MagSpeed: A Novel Method of Vehicle Speed Estimation Through A Single Magnetic Sensor
}

\author{
Yimeng Feng ${ }^{1}$, Guoqiang $\mathrm{Mao}^{2}$, Bo Cheng ${ }^{3}$, Baoqi Huang ${ }^{4}$, Shangbo Wang ${ }^{5}$ and Junliang Chen ${ }^{6}$
}

\begin{abstract}
Internet of Things (IoT) is playing an increasingly important role in Intelligent Transportation Systems (ITS) for real-time sensing and communication. In ITS, the velocity of vehicles provides important information for traffic management. However, the present methods for monitoring vehicle speed have many shortcomings. In this paper, we propose MagSpeed, a novel vehicle speed estimation method based on a small magnetic sensor. The developed magnetic sensor system is wireless, cost-effective, and environmental-friendly. Through modelling of local magnetic field perturbations caused by a moving vehicle, we extract the characteristics of magnetic waveforms for speed estimation. In addition, we compare the performance of the models with other speed estimation algorithms, which shows the superior accuracy of the proposed technique in speed estimation.
\end{abstract}

\section{INTRODUCTION}

Intelligent Transportation System (ITS) has developed rapidly over the last decade. The main purpose of the ITS is to enhance the transportation systems' safety, efficiency, and cost effectiveness. Traffic surveillance provides traffic flow information for ITS through kinds of traffic sensors. And ITS takes the advantage of traffic flow information to improve traffic management. In this paper, we target at the problem of vehicle speed estimation using a single magnetic sensor on the road.

The fine-grained monitoring of vehicle speed plays an important role in ITS. The U.S. National Highway Traffic Safety Administration (NHTSA) reported 37,461 traffic fatalities in 2016, 26\% of which are speed-related. [1] Many traffic surveillance technologies are introduced and have been studies for traffic data collection, like inductive loops [2], video based image processing methods [3], etc. Urban areas have many complex environments, traffic situation estimation needs accurate vehicle speed data.

\footnotetext{
${ }^{1}$ Yimeng Feng is with State Key Laboratory of Networking and Switching Technology, Beijing University of Posts and Telecommunications, China. She is also with School of Electrical and Data Engineering, University of Technology, Sydney, Australia. fyimeng@mail.bupt.edu.cn

${ }^{2}$ Guoqiang Mao is with School of Electrical and Data Engineering, University of Technology, Sydney, Australia. He is also with State Key Laboratory of Integrated Services Networks, Xidian University, China. Guoqiang. Mao@uts. edu. au

${ }^{3}$ Bo Cheng is with State Key Laboratory of Networking and Switching Technology, Beijing University of Posts and Telecommunications, China. chengbo@mail.bupt.edu.cn

${ }^{4}$ Baoqi Huang is with College of Computer Science, Inner Mongolia University, China.

${ }^{5}$ Shangbo Wang is with Institute of Transport and Logistics Studies, The University of Sydney Business School, Australia.

${ }^{6}$ Junliang Chen is with State Key Laboratory of Networking and Switching Technology, Beijing University of Posts and Telecommunications, China.
}

Recently, magnetic sensors are considered for vehicle speed estimation. [4] Current vehicle speed acquisition methods using magnetic sensor often employ more than one magnetic sensors. [5], [6] However, using two or more well separated magnetic sensors for speed estimation, requires these magnetic sensor units to be very well synchronized, which in turn significantly increases both the energy consumption and communication overheads. Both are main considerations for Internet of Things (IoT) devices. Furthermore, the use of two or more magnetic sensor units will significantly increase the size of the IoT device, which also prohibits its widespread use.

In this paper we consider a new approach, which uses a single magnetic sensor to estimate vehicle speed. The underlying idea is to measure the local magnetic field perturbations as vehicles passing by the magnetic sensor. A number of challenges arise in the design of the proposed approach. First, using only one sensor to estimate the result of vehicle speed needs a thorough understanding of metal object's magnetic characteristics. Second, modelling on the local magnetic field perturbation caused by a moving vehicle is essential. Finally, the magnetic sensor readings are noisy and the environment noise needs to be considered and removed to obtain precise results.

This paper presents MagSpeed, a novel vehicle speed estimation method based on a small magnetic sensor. The MagSpeed uses only one magnetic sensor to estimate vehicle velocity by modelling a vehicle as a magnetic dipole. We find that different speeds of a moving magnetic dipole will result in different characteristics of the magnetic waveforms. The filter of raw magnetic data is used for reducing the environmental noise, thus it can give an accurate magnetic output caused by moving vehicle. We give vehicle speed estimation using fitting models. MagSpeed is easy and cost efficient to implement on road. Experiments are conducted in $\mathrm{Xi}$ An, China to establish the effectiveness of the proposed technique.

Specifically, our key contributions are threefold:

- We propose to conduct accurate vehicle speed estimation using only one magnetic sensor. The magnetic sensor is small, cost-effective, and environmental-friendly.

- We present a motion model which illustrates the local magnetic field perturbation caused by a moving vehicle and we give simulation results for the magnetic perturbations caused by a car.

- We develop a vehicle speed estimation technique, MagSpeed, which utilizes filtered magnetic waveform to measure vehicle speeds. Road experiments are con- 
ducted to validate the effectiveness of the proposed technique.

The rest of the paper is organized as follows: Section II briefly reviews related works. Section III describes the design of vehicle speed estimation technique, MagSpeed. Section IV shows the experimental validation of vehicle speed estimation. Finally, Section V concludes the paper.

\section{RELATED WORK}

In this section, we review current methods for vehicle speed estimation, which is divided into two sub-sections. One is general methods of speed acquisition, the other is speed estimation using magnetometers.

\section{A. General methods of speed acquisition}

Many intrusive techniques are used on highways or main roads for speed estimation, such as inductive loops, vision based image processing method, and GPS on smartphone.

Inductive loop detectors that are embedded in the road surface are considered as intrusive methods. [2] These detectors are required to be installed under the road surface, which results in very high installation and maintenance costs.

To reduce installation and maintenance costs, vision based traffic camera systems have been adopted. [3] The data transmission volume is large and the effect is dependent on good weather. In other words, the accuracy of vision based system suffers in bad weather conditions and low illumination and visibility conditions.

Using GPS on smartphone can be a simple way to obtain vehicle speed, it sometimes suffers from urban canyon environment and returns low speed precision.

\section{B. Speed estimation using magnetometers}

Recently, there are a number of researchers considered vehicle detection and speed estimation methods with magnetic sensors. Balid [7] deployed multiple sensors on a road for traffic surveillance. Their work can successfully detect vehicles and estimate various speed of vehicles through a set of magnetic sensors along the road.

\section{Uniform Highly-Distorted Slightly-Distorted Highly-Distorted Uniform}

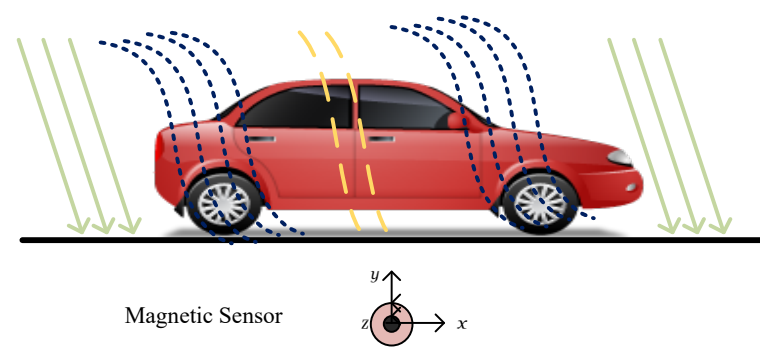

Fig. 1. Magnetic perturbation caused by a vehicle [7]

Obertov in [8] achieved the speed estimation with the accuracy about $90 \%$ through measuring magnetic length using a roadside node with an accelerometer and magnetic sensor.

Taghvaeeyan in [4] proposed applying four magnetic nodes for vehicle speed measurement and vehicle counting, which resulted in good classification rate and average speed detection.

The technique in [5] adopted two magnetic sensors for vehicle speed estimation and a third magnetic sensor for data fusion, which results in speed estimation accurate rate about $80 \%$.

Studies in [9] and [10] proposed algorithms for speed estimation using two magnetometers. It is worth noting that these methods need to estimate average speed based on the number of passing vehicles over time.

Vehicle detection and classification through an improved support vector machine classifier was proposed in [11] using magnetic sensors. They use magnetic signatures to distinguish different kinds of vehicles, such as heavy tracked, tracked, and light-wheeled vehicles. However, the experiment setup is expensive, which required an enormous amount of road work as well as it requires high processing capability.

Marshall in [12] first introduced metal's magnetic effect of vehicle body and simulated a scenario to detect a vehicle using magnetic sensors. Chueng in [13], [14] developed a feature selection model for vehicle classification using a single magnetic sensor in which 17 features were initially identified and extracted, and 10 optimal features were eventually selected for classification.

There are also work on using combination of magnetic sensors for vehicle information acquisition. Studies in [15], [16], [17] propose vehicle classification based on machine learning of the signal waveforms.

Obtaining the vehicle speed is becoming more and more crucial in supporting real-time traffic management. In the aforementioned studies, speed was estimated using two or more magnetic sensors.

Our work is different from the previous studies in that we investigate a single magnetic sensor approach for vehicle speed estimation without damage to the road.

\section{Design Of MAGSPEED}

In this section, we present the design of our proposed system, MagSpeed, which estimates vehicle speed through measuring and modelling local magnetic field perturbation caused by moving vehicles. The deployment of MagSpeed only depends on a tiny magnetic sensor. We first explain MagSpeed from the working mechanism of metal objects' magnetic characteristics, then describe the motion process of moving vehicle, and finally illustrate the filter model for removing background noise.

\section{A. Magnetic dipole Model}

For a vehicle with a certain speed passing on a road, it leads to perturbations of the local magnetic fields. The vehicle can be seen as a magnetic dipole [18]. Fig. 1 illustrates the magnetic distribution of the magnetic flux lines when the earth's magnetic field is temp oratory changed by 
a vehicle, of which the wheels are distorted highly and the body is slightly distorted. For a magnetic dipole, its magnetic field can be expressed as (1) [19], [20]:

$$
\boldsymbol{B}=\left[\begin{array}{ccc}
3 x^{2}-r^{2} & 3 x y & 3 x z \\
3 x y & 3 y^{2}-r^{2} & 3 y z \\
3 x z & 3 y z & 3 z^{2}-r^{2}
\end{array}\right] \bullet \frac{u_{0} \boldsymbol{m}}{4 \pi r^{5}}
$$

where $m$ is the magnetic moment of a dipole, $u_{0}$ is magnetic permeability, $r$ is the distance between the measurement position and the magnetic dipole, with $r^{2}=x^{2}+y^{2}+z^{2}$. The tensor notation of the equation in the orthogonal coordinate system will take the following form with the expansion of magnetic vector being $\boldsymbol{m}=\left(\begin{array}{lll}\boldsymbol{m}_{\boldsymbol{x}} & \boldsymbol{m}_{\boldsymbol{y}} & \boldsymbol{m}_{\boldsymbol{z}}\end{array}\right)$ :

$\boldsymbol{B}=\left[\begin{array}{ccc}3 x^{2}-r^{2} & 3 x y & 3 x z \\ 3 x y & 3 y^{2}-r^{2} & 3 y z \\ 3 x z & 3 y z & 3 z^{2}-r^{2}\end{array}\right] \bullet\left[\begin{array}{c}m_{x} \\ m_{y} \\ m_{z}\end{array}\right] \cdot \frac{u_{0}}{4 \pi r^{5}}$

where $m_{x}$ is the component of the magnetic moment on the $\mathrm{x}$-axis, $m_{y}$ is the component of the magnetic moment on the y-axis and $m_{z}$ is the component of the magnetic moment on the z-axis, respectively, $\bullet$ and means dot product. It follows from (2) that:

$$
\begin{aligned}
& B_{x}=\left(\left(3 x^{2}-r^{2}\right) \cdot m_{x}+3 x y \cdot m_{y}+3 x z \cdot m_{z}\right) \cdot \frac{u_{0}}{4 \pi r^{5}} \\
& B_{y}=\left(3 x y \cdot m_{x}+\left(3 y^{2}-r^{2}\right) \cdot m_{y}+3 y z \cdot m_{z}\right) \cdot \frac{u_{0}}{4 \pi r^{5}} \\
& B_{z}=\left(3 x z \cdot m_{x}+3 y z \cdot m_{y}+\left(3 z^{2}-r^{2}\right) \cdot m_{z}\right) \cdot \frac{u_{0}}{4 \pi r^{5}}
\end{aligned}
$$

For the magnetic value of $x$ axis, we expand the value of $r$ and simplify the expression of $B_{x}$ as follows:

$$
\begin{gathered}
B_{x}=\left(\left(2 x^{2}-y^{2}-z^{2}\right) \cdot m_{x}+3 x y \cdot m_{y}+3 x z \cdot m_{z}\right) \\
\cdot \frac{u_{0}}{4 \pi\left(x^{2}+y^{2}+z^{2}\right)^{\frac{5}{2}}}
\end{gathered}
$$

Because the exact movement direction and value of magnetic moment are unknown, we set default $m=(1,1,1)$ for simulation. [21], [22], [19], [23]. Fig. 2 gives the magnetic waveform corresponding to different speed of a magnetic dipole on $x$ axis, which varies between $20 \mathrm{~km} / \mathrm{h}$ to $50 \mathrm{~km} / \mathrm{h}$. As can be seen from the figure, with different vehicle speeds, the period for detected magnetic distortion is changing over time.

\section{B. Moving Vehicle Motion Model}

Three-dimensional Cartesian coordinate system is set up from the view of the magnetic sensor, which is the original point $(0,0,0)$. For a moving vehicle with a speed of $v_{x}\left(v_{x}>\right.$ $0)$, and an initial position $\left(x_{0}, y_{0}, z_{0}\right)$, driving along the $\mathrm{x}$ axis. The initial value of $x_{0}<0$, the initial value of $y_{0}$ is zero and the initial value of $z_{0}$ is non-negative and is small. After

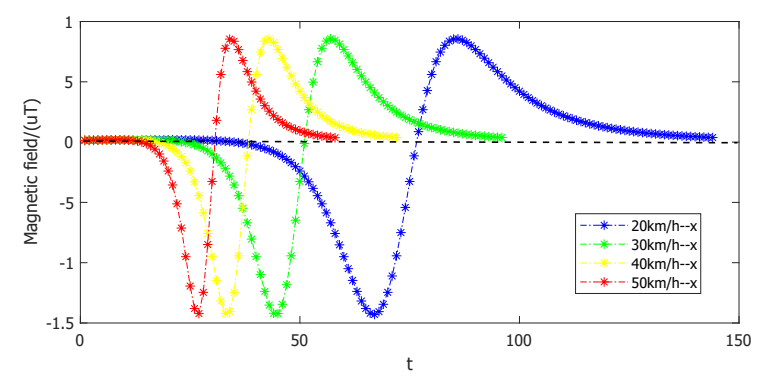

Fig. 2. The simulation of $\mathrm{x}$-axis from $20 \mathrm{~km} / \mathrm{h}$ to $50 \mathrm{~km} / \mathrm{h}$

a period of time $t(t>0)$, the vehicle's location becomes $\left(x_{0}+v_{x} \cdot t, y_{0}, z_{0}\right)$.

Then the function for $B_{x}$ of $t$ is given like:

$$
\begin{gathered}
f(t)=2\left(x_{0}+v_{x} \cdot t\right)^{2} \cdot m_{x} \cdot \frac{u_{0}}{4 \pi\left(\left(x_{0}+v_{x} \cdot t\right)^{2}+D\right)^{\frac{5}{2}}} \\
+3 v_{x} \cdot t \cdot y_{0} \cdot m_{y} \cdot \frac{u_{0}}{4 \pi\left(\left(x_{0}+v_{x} \cdot t\right)^{2}+D\right)^{\frac{5}{2}}} \\
+3 v_{x} \cdot t \cdot z_{0} \cdot m_{z} \cdot \frac{u_{0}}{4 \pi\left(\left(x_{0}+v_{x} \cdot t\right)^{2}+D\right)^{\frac{5}{2}}} \\
+C \cdot \frac{u_{0}}{4 \pi\left(\left(x_{0}+v_{x} \cdot t\right)^{2}+D\right)^{\frac{5}{2}}} \\
=\frac{\mathrm{A} *\left(x_{0}+v_{x} \cdot t\right)^{2}+B * v_{x} \cdot t+C}{\left(x_{0}+v_{x} \cdot t\right)^{5}+D}
\end{gathered}
$$

where $A=2 \frac{m_{x} \cdot u_{0}}{4 \pi}$,

$$
\begin{aligned}
& B=3 \frac{z_{0} \cdot m_{z} \cdot u_{0}}{4 \pi}, \\
& C=\frac{3 x_{0} \cdot z_{0} \cdot m_{z} \cdot u_{0}-z_{0}^{2} \cdot m_{x} \cdot u_{0}}{4 \pi}, \\
& D=\left(\left(x_{0}+v_{x} \cdot t\right)^{2}+z_{0}^{2}\right)^{\frac{5}{2}}-\left(x_{0}+v_{x} \cdot t\right)^{5}
\end{aligned}
$$

Since $z_{0}$ can be assumed to be small, the values of $B$ and $C$ are also small and can be neglected. Thus, an approximation can be applied.

$$
B_{x} \approx \frac{A *\left(x_{0}+v_{x} \cdot t\right)^{2}}{\left(x_{0}+v_{x} \cdot t\right)^{5}+D}
$$

Let $f(t)=B_{x}$,

$$
f(t)=\frac{A *\left(x_{0}+v_{x} \cdot t\right)^{2}}{\left(x_{0}+v_{x} \cdot t\right)^{5}+D}
$$

The denominator in (9) is polynomial and is hard to analyze fifth root at polynomial. Let the reciprocal of $f(t)$ be $g(t)=\frac{1}{f(t)}$, then

$$
g(t)=\frac{\left(x_{0}+v_{x} \cdot t\right)^{5}+D}{A *\left(x_{0}+v_{x} \cdot t\right)^{2}}
$$


Meanwhile, letting $v_{x} \cdot t=x . x$ reflects the change of positions with a speed $v_{x}$ as time $t$ passes. Because $v_{x}>0$ and $t>0$, so $x>0$, and we have

$$
\begin{gathered}
g(x)=\frac{1}{A}\left(x_{0}+x\right)^{3}+\frac{D}{A\left(x_{0}+x\right)^{2}} \\
=\frac{1}{A} x^{3}+\frac{3 x_{0}}{A} x^{2}+\frac{3 x_{0}}{A} x+\frac{x_{0}{ }^{3}}{A}+\frac{D}{A\left(x_{0}+x\right)^{2}}
\end{gathered}
$$

As the value of $x(x>0)$ increases, $\frac{1}{A} x^{3}+\frac{3 x_{0}}{A} x^{2}+$ $\frac{3 x_{0}}{A} x+\frac{x_{0}^{3}}{A}$ increases, and $\frac{D}{A\left(x_{0}+x\right)^{2}}$ decreases. There is a turning point that reaches the local minimum value for $g(x)$ is at $x=\sqrt[5]{-D}-x_{0}$, which allows $\frac{1}{A} x^{3}+\frac{3 x_{0}}{A} x^{2}+\frac{3 x_{0}}{A} x+$ $\frac{x_{0}^{3}}{A}+\frac{D}{A\left(x_{0}+x\right)^{2}}=0$.

If $x<\sqrt[5]{-D}-x_{0}$, the dominating term for $g(x)$ is $\frac{D}{A\left(x_{0}+x\right)^{2}}$, thus $g(x)=\frac{D}{A\left(x_{0}+x\right)^{2}}+\varepsilon_{1}, \varepsilon_{1}$ is system error.

If $x=\sqrt[5]{-D}-x_{0}, g(x)=0$.

If $x>\sqrt[5]{-D}-x_{0}$, the dominating term for $g(x)$ is $\frac{1}{A}\left(x_{0}+\right.$ $x)^{3}$,thus $g(x)=\frac{1}{A}\left(x_{0}+x\right)^{3}+\varepsilon_{2}, \varepsilon_{2}$ is system error.

$$
g(x)= \begin{cases}\frac{D}{A\left(x_{0}+x\right)^{2}}+\varepsilon_{1} & x<\sqrt[5]{-D}-x_{0} \\ 0 & x=\sqrt[5]{-D}-x_{0} \\ \frac{1}{A}\left(x_{0}+x\right)^{3}+\varepsilon_{2} & x>\sqrt[5]{-D}-x_{0}\end{cases}
$$

$x_{0}, v_{x}$ does not change with time $t$. Through the analysis of $g(x)$, there are four approximations models of $f(t)$ given as follows based on the above discussions:

Model 1: power form

$$
f_{1}(t)=a t^{b}+c+\varepsilon_{1}
$$

Model 2: polynomial form

$$
f_{2}(t)=a_{0}+a_{1} t+a_{2} t^{2}+a_{3} t^{3}+\ldots+a_{n} t^{n}+\varepsilon_{2}
$$

Model 3: rational form

$$
f_{3}(t)=\frac{p_{1}}{t^{3}+q_{1} t^{2}+q_{2} t+q_{3}}+\varepsilon_{3}
$$

Model 4: combination of polynomial form and rational form

$$
f_{4}(t)=\lambda_{1} t^{3}+\lambda_{2} t^{2}+\lambda_{3} t+\lambda_{4}+\lambda_{5} \cdot\left(t^{(-3)}\right)+\varepsilon_{4}
$$

Now the relationships between time and the magnetic field perturbation caused by a moving vehicle are given as aforementioned. If we combine the models with real time data $t$, the parameters above can be calculated correspondingly. There is a value $t_{0}$ when $B_{x}=0$, which is the point which allows $f(t)=0$. For different speeds, $t_{0}$ s are different, which reflects the interval between the beginning of $\mathrm{x}$-axis and the time at $B_{x}=0$. Given the different $t_{0} \mathrm{~s}$, it will reflect the real driving speed of the vehicles respectively, which outputs the estimation results of vehicle speed. In addition, the $t_{0} \mathrm{~s}$ are obtained from real traffic data and preprocessed from noise. So signal processing techniques and filter techniques are applied in the next section. Experimental results for the models are given in section IV.

\section{Signal Processing Model}

Measurements in real road condition are full of background noise and are affected by earth's magnetic field. [18] It makes the signals hard to distinguish the passing vehicles. So a low pass filter is used to make the waveforms pure and clear. [11]

The earth's magnetic value are $\left(B_{x 0}, B_{y 0}, B_{z 0}\right)$, and $\left(X_{i}, Y_{i}, Z_{i}\right)$ are the values recorded by the magnetic sensor when a vehicle is passing. $\left(X_{i}, Y_{i}, Z_{i}\right)$ is the The perturbation caused by a passing vehicle is $\left(B_{x}, B_{y}, B_{z}\right)$, then

$$
\left(B_{x}, B_{y}, B_{z}\right)=\left(X_{i}-B_{x 0}, Y_{i}-B_{y 0}, Z_{i}-B_{z 0}\right)
$$

Fig. 3 shows the waveforms of $\left(X_{i}, Y_{i}, Z_{i}\right)$ for a small vehicle at the speed of $30 \mathrm{~km} / \mathrm{h}$. The vertical axis $B(\mu T)$ reflects the perturbation caused by the small vehicle. The horizontal axis $t$ reflects time with the sampling frequency of a magnetic sensor.

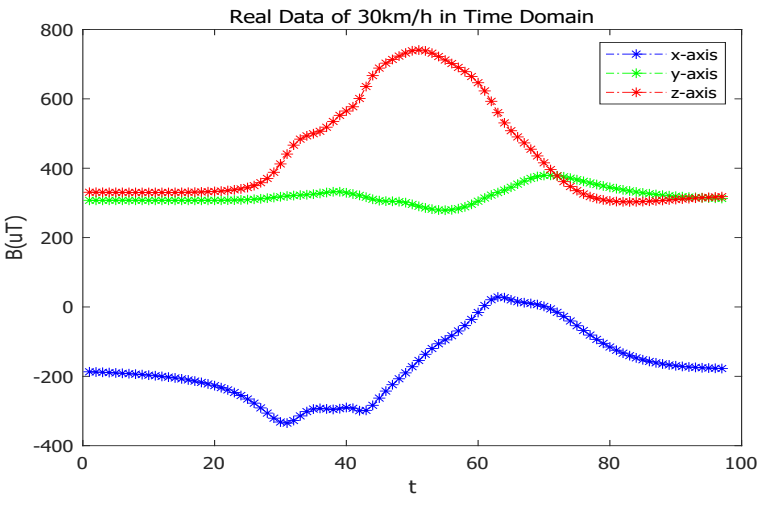

Fig. 3. Real magnetic perturbtation before the filter model

There are lots of noises in the real environment, besides reduce the $\left(B_{x 0}, B_{y 0}, B_{z 0}\right)$, we apply Fast Fourier Transform (FFT) and use a low pass filter for $\left(B_{x}, B_{y}, B_{z}\right)$ to eliminate noise effect. So after the FFT signal processing, Fig. 4 shows the filtered and normalized waveforms for a vehicle at the speed of $30 \mathrm{~km} / \mathrm{h}$.

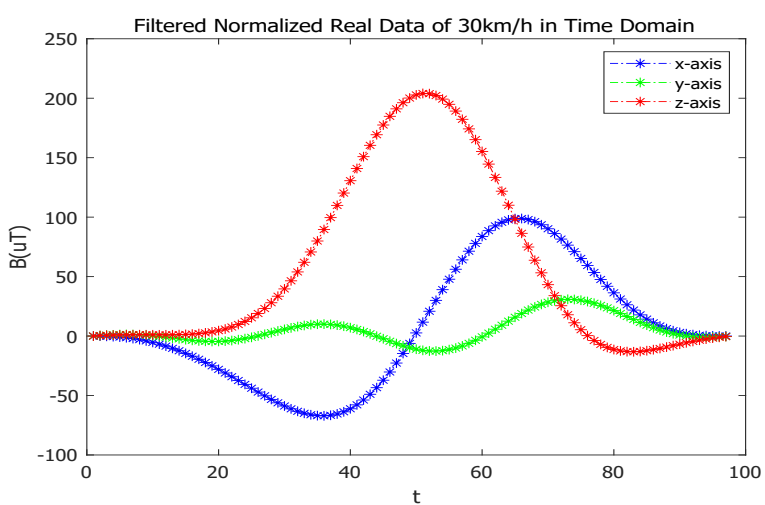

Fig. 4. Real magnetic perturbation after the filter model

Through the signal processing model, it is visual to separate $t_{0}$ for each speed's waveform. The missing data 
issue is also not negligible, so we conduct 5 times experiment for each speed and obtain the mean value of $t_{0} \mathrm{~s}$ to eliminate random errors. In the following section, we explain our experiments on road with a car driving in different speeds.

\section{EXPERIMENTAL VALIDATION}

In this section, we present the evaluation of our speed estimation system, MagSpeed, in real driving environments in Xian, China.

\section{A. Experiment Setup}

We evaluate our speed estimation system, MagSpeed, in real driving environments with a single magnetic sensor on the road. Fig. 5 shows an experiment setup on a target road. The sensor in the experiment is RM3100 magnetic sensor, which outputs magnetic vibration in three axis. The size of the chip is only as large as a coin and the overall size of the sensor is tiny, which is portable for traffic surveillance. We also put a camera on roadside to record the overall driving period. The setup is mainly aimed at urban roads with low speeds. Therefore, for each speed of $15 \mathrm{~km} / \mathrm{h}, 20 \mathrm{~km} / \mathrm{h}, 25$ $\mathrm{km} / \mathrm{h}, 30 \mathrm{~km} / \mathrm{h}, 35 \mathrm{~km} / \mathrm{h}, 40 \mathrm{~km} / \mathrm{h}, 45 \mathrm{~km} / \mathrm{h}$, we drive five times to ensure we have obtained accurate magnetic data.

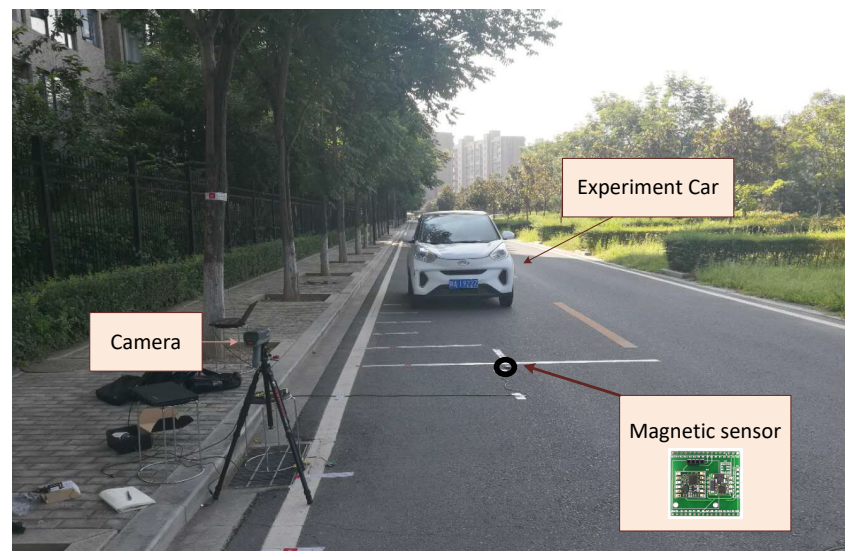

Fig. 5. Experiment setup

\section{B. Experimental Results}

After combining real world data with MagSpeed, we receive the estimated values of each fitting models based on the discussion in section III. Given the time of driving vehicle, parameters are calculated by MATLAB R2018 on windows 10 as follows.

For power form, $f_{1}(t)=a t^{b}+c+\varepsilon_{1}$. Coefficients (with 95\% confidence bounds): $a=16.42, b=-0.8424, c=$ -0.01758 .

For polynomial form, $f_{2}(t)=a_{0}+a_{1} t+a_{2} t^{2}+a_{3} t^{3}+$ $\ldots+a_{n} t^{n}+\varepsilon_{2}$. Coefficients (with $95 \%$ confidence bounds): $a_{0}=102.3, a_{1}=-275.2, a_{2}=325.9, a_{3}=-136.2$.

For rational form, $f_{3}(t)=\frac{p_{1}}{t^{3}+q_{1} t^{2}+q_{2} t+q_{3}}+\varepsilon_{3}$. Coefficients (with 95\% confidence bounds): $p 1=10.51, q 1=$ $-1.819, q 2=1.585, q 3=-0.1058$.
For combination form, $f_{4}(t)=\lambda_{1} t^{3}+\lambda_{2} t^{2}+\lambda_{3} t+\lambda_{4}+$ $\lambda_{5} \cdot\left(t^{(-3)}\right)+\varepsilon_{4}$. Coefficients (with $95 \%$ confidence bounds): $\lambda_{1}=37.36, \lambda_{2}=-77.11, \lambda_{3}=27.48, \lambda_{4}=27.56, \lambda_{5}=$ 0.4019 .

\section{Discussion}

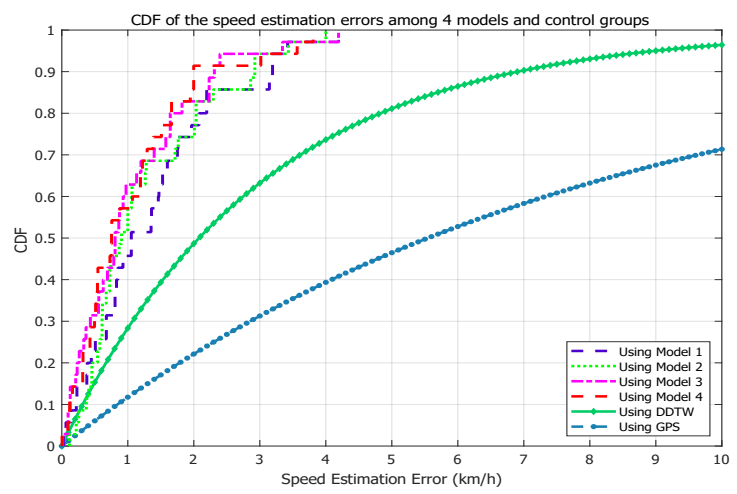

Fig. 6. CDF of the speed estimation errors among 4 models and control groups

Here we discuss the four proposed models with other work. Fig. 6 plots the cumulative distribution function (CDF) of the speed estimation errors $(\mathrm{km} / \mathrm{h})$ using the four proposed models, Derivative Dynamic Time Warping (DDTW) [24] and Localization algorithms. The results explain that we achieve high speed estimation performance in all four proposed models. For example, $80 \%$ of estimation errors are lower than $2 \mathrm{~km} / \mathrm{h}$ if using the four proposed models, and $50 \%$ of estimation errors are less than $1 \mathrm{~km} / \mathrm{h}$. In addition, only $10 \%$ of estimated speed errors in model 4 exceeds $2 \mathrm{~km} / \mathrm{h}$ and almost all fitting models achieve high accuracy within $5 \mathrm{~km} / \mathrm{h}$. Thus, the proposed fitting models are robust for vehicle speed estimation. In the meantime, we compare the four proposed models with DDTW (Derivative Dynamic Time Warping) and GPS. From Fig. 6, it can be seen that the fitting models outperforms GPS in speed estimation. Compared with DDTW, the fitting models still remain high precision. For example, 90\% of DDTW's estimation errors are less than $7 \mathrm{~km} / \mathrm{h}$. By contrast, all the estimation errors of the proposed models are lower than $5 \mathrm{~km} / \mathrm{h}$.

\section{Vehicle Speed Estimation Error Analysis}

In this part, we give the histograms of our four models to show the distribution of errors. As in Fig 7, through observation of the four models' estimation error distributions, we can see the model that has less error and centered distribution is Model 4, which is the closest to the deviation result in Section III.

Thus, for model 4 , we achieve an error distribution result with $\mu=0.0030, \sigma^{2}=1.4356$, which has the mean of 0.0030 , variance of 2.061 . The overall vehicle speed estimation accuracy is $96.7 \%$. For all models, the average vehicle speed estimation accuracy is $92.5 \%$. 


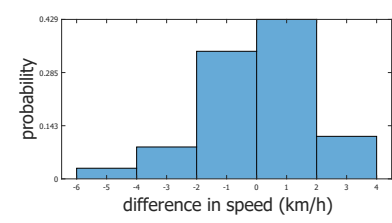

(a) Model 1

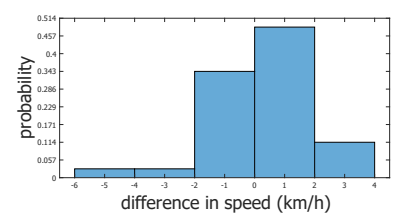

(c) Model 3

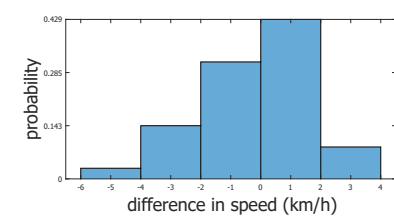

(b) Model 2

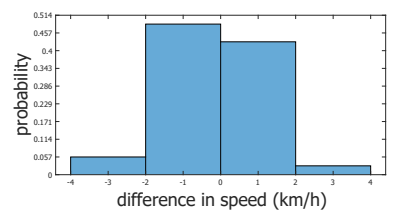

(d) Model 4
Fig. 7. Histograms of four models

\section{Conclusions}

In this paper, we address the problem of performing accurate vehicle speed estimation in urban environments through a single magnetic sensor. In particular, we develop a vehicle speed estimation technique, MagSpeed, which utilizes normalized and filtered magnetic waveforms to measure vehicle speeds. Also, road experiments are conducted to validate the effectiveness of the proposed technique. The magnetic sensor in our experiment is tiny, cost-effective, and environmental-friendly. While, sometimes the magnetic vibrations of the earth is not stable in different places as well as the adjacent lanes have effects on the measurement. In addition, it is part of our future work to combine with other sensor information to improve the performance of finegrained speed estimation. We will add analysis with multiple types of vehicles in the future through modelling analysis and real road test experiments.

\section{ACKNOWLEDGMENT}

This work was supported in part by the National Key Research and Development Program of China (2017YFB1400603). The authors would like to thank the anonymous reviewers for their comments. The authors would like to thank the effort of the students from Xidian University for the road experiment assistance.

\section{REFERENCES}

[1] N. H. T. S. A. Washington DC, "National center for statistics and analysis." (2018, March, revised) Speeding: 2016 data (Traffic Safety Facts. DOT HS 812 480).

[2] C. Sun and S. G. Ritchie, "Individual vehicle speed estimation using single loop inductive waveforms," Journal of Transportation Engineering, vol. 125, no. 6, pp. 531-538, 1999.

[3] T. N. Schoepflin and D. J. Dailey, "Dynamic camera calibration of roadside traffic management cameras for vehicle speed estimation," IEEE Transactions on Intelligent Transportation Systems, vol. 4, no. 2, pp. 90-98, 2003.

[4] S. Taghvaeeyan and R. Rajamani, "Portable roadside sensors for vehicle counting, classification, and speed measurement," IEEE Transactions on Intelligent Transportation Systems, vol. 15, no. 1, pp. 7383, 2014.

[5] Q. Wei and B. Yang, "Adaptable vehicle detection and speed estimation for changeable urban traffic with anisotropic magnetoresistive sensors," IEEE Sensors Journal, vol. 17, no. 7, pp. 2021-2028, 2017.
[6] W. Balid, H. Tafish, and H. H. Refai, "Development of portable wireless sensor network system for real-time traffic surveillance," in 2015 IEEE 18th International Conference on Intelligent Transportation Systems, Conference Proceedings, pp. 1630-1637.

[7] _ - "Intelligent vehicle counting and classification sensor for realtime traffic surveillance," IEEE Transactions on Intelligent Transportation Systems, vol. 19, no. 6, pp. 1784-1794, 2018.

[8] D. Obertov, V. Bardov, and B. Andrievsky, "Vehicle speed estimation using roadside sensors," in 2014 6th International Congress on Ultra Modern Telecommunications and Control Systems and Workshops (ICUMT), Conference Proceedings, pp. 111-117.

[9] H. Li, H. Dong, L. Jia, D. Xu, and Y. Qin, "Some practical vehicle speed estimation methods by a single traffic magnetic sensor," in 2011 14th International IEEE Conference on Intelligent Transportation Systems (ITSC). IEEE, Conference Proceedings, pp. 1566-1573.

[10] D. Xiaoyong, H. Zhongwei, P. Zhang, and G. Jifu, "Vehicle class composition identification based mean speed estimation algorithm using single magnetic sensor," Journal of Transportation Systems Engineering and Information Technology, vol. 10, no. 5, pp. 35-39, 2010.

[11] J. Lan, Y. Xiang, L. Wang, and Y. Shi, "Vehicle detection and classification by measuring and processing magnetic signal," Measurement, vol. 44, no. 1 , pp. 174-180, 2011.

[12] S. V. Marshall, "Vehicle detection using a magnetic field sensor," IEEE Transactions on Vehicular Technology, vol. 27, no. 2, pp. 65-68, 1978.

[13] S. Cheung, S. Coleri, B. Dundar, S. Ganesh, C.-W. Tan, and P. Varaiya, "Traffic measurement and vehicle classification with single magnetic sensor," Transportation research record: journal of the transportation research board, no. 1917, pp. 173-181, 2005.

[14] S. Y. Cheung, S. C. Ergen, and P. Varaiya, "Traffic surveillance with wireless magnetic sensors," in Proceedings of the 12th ITS world congress, vol. 1917, Conference Proceedings, p. 173181.

[15] Q. Wang, J. Zheng, H. Xu, B. Xu, and R. Chen, "Roadside magnetic sensor system for vehicle detection in urban environments," IEEE Transactions on Intelligent Transportation Systems, vol. 19, no. 5, pp. 1365-1374, 2018.

[16] D. Kleyko, R. Hostettler, W. Birk, and E. Osipov, "Comparison of machine learning techniques for vehicle classification using road side sensors," in 2015 IEEE 18th International Conference on Intelligent Transportation Systems, Conference Proceedings, pp. 572-577.

[17] H. Li, H. Dong, L. Jia, D. Xu, and Y. Qin, "Some practical vehicle speed estimation methods by a single traffic magnetic sensor," in Intelligent Transportation Systems (ITSC), 2011 14th International IEEE Conference on. IEEE, Conference Proceedings, pp. 1566-1573.

[18] S. Gontarz and Szulim, "Use of magnetic monitoring of vehicles for proactive strategy development," Transportation Research Part C: Emerging Technologies, vol. 52, pp. 102-115, 2015. [Online]. Available: http://www.sciencedirect.com/science/article/pii/ S0968090X1400357X

[19] J. Xie, C. Qin, X. Zhou, L. Huang, X. Han, M. Wang, and L. Li, "The simulations and experiments of the electromagnetic tracking system based on magnetic dipole model," IEEE Transactions on Applied Superconductivity, vol. 24, no. 3, pp. 1-4, 2014.

[20] N. WahlstrÃ $\llbracket \mathrm{m}$ and F. Gustafsson, "Magnetometer modeling and validation for tracking metallic targets," IEEE Transactions on Signal Processing, vol. 62, no. 3, pp. 545-556, 2014.

[21] Q. Zhou, G. Tong, B. Li, and X. Yuan, "A practicable method for ferromagnetic object moving direction identification," IEEE Transactions on Magnetics, vol. 48, no. 8, pp. 2340-2345, 2012.

[22] S. Gontarz and S. Radkowski, "Impact of various factors on relationships between stress and eigen magnetic field in a steel specimen," IEEE Transactions on Magnetics, vol. 48, no. 3, pp. 1143-1154, 2012.

[23] Y. Ren, C. Hu, S. Xiang, and Z. Feng, "Magnetic dipole model in the near-field," in 2015 IEEE International Conference on Information and Automation, Conference Proceedings, pp. 1085-1089.

[24] G. Chandrasekaran, T. Vu, A. Varshavsky, M. Gruteser, R. P. Martin, J. Yang, and Y. Chen, "Tracking vehicular speed variations by warping mobile phone signal strengths," in 2011 IEEE International Conference on Pervasive Computing and Communications (PerCom). IEEE, Conference Proceedings, pp. 213-221. 